\title{
NMR relaxation rate of a quantum spin chain with an impurity
}

\author{
A.A. Zvyagin \\ Max-Planck-Institut für Physik komplexer Systeme, 38 Nöthnitzer Str., Dresden D-01187, Germany \\ B. Verkin Institute for Low Temperature Physics and Engineering of the National Academy of Sciences of Ukraine \\ 47 Lenin Ave., Kharkov 61103, Ukraine \\ E-mail: zvyagin@ilt.kharkov.ua
}

Received March 2, 2012

\begin{abstract}
The relaxation rate of the nuclear magnetic resonance is calculated exactly for a model spin-1/2 chain with the magnetic impurity. It is shown that the deviations of the impurity's coupling to the chain and the effective impurity's $g$-factor from the values of the host chain yield special features in the temperature and magnetic field behavior for the relaxation rate.
\end{abstract}

PACS: 75.10.Jm Quantized spin models, including quantum spin frustration;

75.10.Pq Spin chain models;

76.60.-k Nuclear magnetic resonance and relaxation.

Keywords: nuclear magnetic resonance, spin chain, magnetic impurity.

The interest in quasi-one-dimensional quantum systems has substantially grown recently due to progress in synthesizing materials with quasi-one-dimensional electron subsystems, as oxides of transition metals, or organic compounds. On the other hand, theory of one-dimensional quantum spin models permits to calculate in many cases exactly their characteristics. Moreover, in the last decade one-dimensional quantum spin models are often used to realize the ideas of the quantum information. Also, interesting phenomena in those systems, like anisotropic heat/spin transport, or a large anisotropy of magnetic interactions, have a perspective to be realized in technological applications.

The one-dimensional model with antiferromagnetic interactions between neighboring spins $1 / 2$ is one of the best studied models of the modern physics. However, in real low-dimensional spin systems we often deal with a system with imperfections, e.g., impurities. Due to peculiarities in the one-dimensional density of states, impurities can drastically change the behavior of one-dimensional spin systems, in particular, the presence of impurities in an electron chain can yield a localization of eigenstates. Hence to take into account the effect of impurities in one-dimensional systems we need a non-perturbative approach. It is possible to realize in the framework of integrable spin models with impurities [1]. Many static characteristics, like the magnetic susceptibility and specific heat were exactly calculated for spin chain models. However, the calculation of dynamical characteristics is more difficult, and only few exact results were obtained for spin chains with impurities.

Important low-energy dynamical characteristics of the spin system can be measured in the nuclear magnetic resonance (NMR) experiments. NMR experiments on quasione-dimensional magnets have been performed since their first realization in 70s up to the present time [2]. Their high sensitivity to the static and dynamic local hyperfine fields allows to perform detailed studies of magnetic impurities.

We start with the considertion of the Hamiltonian of the inhomogeneous semi-infinite spin $1 / 2$ chain with the magnetic impurity situated at the edge of the chain [1]

$$
\begin{gathered}
\mathcal{H}=- \\
-J \sum_{n=1}^{N-1}\left(S_{n}^{x} S_{n+1}^{x}+S_{n}^{y} S_{n+1}^{y}\right)-g \mu_{B} H \sum_{n=1}^{N} S_{n}^{z}- \\
-J^{\prime}\left(S_{0}^{x} S_{1}^{x}+S_{0}^{y} S_{1}^{y}\right)-g^{\prime} \mu_{B} H S_{0}^{z},
\end{gathered}
$$

where $S_{n}^{x, y, z}$ are the operators of the spin-1/2 projections at the site $n$ (impurity is situated at $n=0), J\left(J^{\prime}\right)$ describes the strength of spin-spin interactions between neighboring spins in the host chain (between the chain and impurity situated at the site 0$), g\left(g^{\prime}\right)$ denotes the effective $g$-factor for the host chain (impurity), $\mu_{B}$ is Bohr's magneton, $H$ is the external magnetic field, and $N$ is the number of sites in the chain (we consider $N$ odd; in the thermodynamic limit we take $N \rightarrow \infty$ ). The local renorma- 
lization of some exchange couplings between spins and/or $g$-factors of some spins in a spin chain can be caused in real magnetic systems, e.g., by substitution of ligands, like $\mathrm{Ge}$ instead of $\mathrm{Si}$ in a spin-1/2 chain compound $\mathrm{BaCu}_{2}\left(\mathrm{Si}_{1-x} \mathrm{Ge}_{x}\right)_{2} \mathrm{O}_{7}$ [3], and $\mathrm{Ca}$ instead of $\mathrm{Sr}$ in $\mathrm{Sr}_{1-x} \mathrm{Ca}_{x} \mathrm{CuO}_{2}$ [4].

It is known [1] that thermodynamic properties of the host chain do not depend on the sign of $J$. After the Jordan-Wigner transformation [5]

$$
\begin{gathered}
2 S_{n}^{z}=1-2 a_{n}^{\dagger} a_{n}, \\
S_{n}^{+}=\prod_{m<n}\left(1-2 a_{m}^{\dagger} a_{m}\right) a_{n}, \\
S_{n}^{-}=a_{n}^{\dagger} \prod_{m<n}\left(1-2 a_{m}^{\dagger} a_{m}\right),
\end{gathered}
$$

where $a_{n}$ and $a_{n}^{\dagger}$ are Fermi operators of creation and destruction, the Hamiltonian Eq. (1) is exactly transformed to the quadratic form of Fermi operators

$$
\begin{gathered}
\mathcal{H}=-\frac{J}{2} \sum_{n=1}^{N-1}\left(a_{n}^{\dagger} a_{n+1}+a_{n+1}^{\dagger} a_{n}\right)+g \mu_{B} H \sum_{n=1}^{N} a_{n}^{\dagger} a_{n}- \\
-\frac{J^{\prime}}{2}\left(a_{0}^{\dagger} a_{1}+a_{1}^{\dagger} a_{0}\right)+g^{\prime} \mu_{B} H a_{0}^{\dagger} a_{0}-\frac{\mu_{B} H}{2}\left(N g+g^{\prime}\right) .
\end{gathered}
$$

This quadratic form can be diagonalized by the unitary transformation

$$
a_{n}=\sum_{\lambda} u_{n, \lambda} a_{\lambda}, n=0,1, \ldots, N
$$

For the considered semi-infinite chain with the impurity in the thermodynamic limit we can exactly write the eigenfunctions $u_{n, \lambda}$ in the co-ordinate representation and eigenvalues $\varepsilon_{\lambda}$. There are two kinds of contributions. The first one is related to the extended states (continuous spectrum) of the spin chain

$$
\varepsilon_{k}=g \mu_{B} H-J \cos (k),
$$

with the wave functions [6]

$$
\begin{aligned}
u_{n, k}= & \sqrt{\frac{2}{\pi A_{k}}}(2[x+\cos (k)] \sin (n k)- \\
& \left.-I^{2} \sin [(n-1) k]\right), n \neq 0,
\end{aligned}
$$

and

$$
u_{0, k}=\sqrt{\frac{2}{\pi A_{k}}} I \sin (k),
$$

where

$$
A_{k}=[4 x+\cos (k)]^{2}+I^{4}-4 I^{2} \cos (k)[x+\cos (k)],
$$

$I=J^{\prime} / J$ measures the relative strength of the impurityhost coupling, $x=\mu_{B}\left(g^{\prime}-g\right) H / J$ measures the relative difference between the local magnetic moments of the impurity and the host due to the difference of the effective $g$-factors. Extended states, associated with the continuous spectrum, are present, naturally, in the homogeneous spin chain, too, and the impurity renormalizes the parameters of extended states. For $I^{2}>2 \mp 2 x$ local levels, caused by the impurity, are split off. These impurity local levels have the energies

$$
\varepsilon_{1,2}=g \mu_{B} H-J\left(r_{1,2}^{2}+1\right) / 2 r_{1,2},
$$

with the wave functions in the co-ordinate representation [6]

$$
u_{n, 1,2}=I r_{1,2}^{n}\left[\frac{\left(1-r_{1,2}^{2}\right)}{\left(1+\left[I^{2}-1\right] r_{1,2}^{2}\right)}\right]^{1 / 2} \text {, }
$$

and

$$
u_{0,1,2}=\left[\frac{\left(1-r_{1,2}^{2}\right)}{\left(1+\left[I^{2}-1\right] r_{1,2}^{2}\right)}\right]^{1 / 2},
$$

where

$$
r_{1,2}=\frac{\left(x \pm \sqrt{x^{2}+I^{2}-1}\right)}{\left(I^{2}-1\right)} .
$$

For $I=1$, i.e., for the situation, in which the impurity differs from the host chain only by the value of the $g$-factor, we have only one local level with $r=-1 / 2 x$ and the energy $\varepsilon=g \mu_{B} H+J\left(1+4 x^{2}\right) / 4 x$. Obviously, local levels exist for $\left|r_{1,2}\right|<1,\left(|r|<1\right.$ for $\left.J^{\prime}= \pm J\right)$, i.e., their wave functions decay with the growth of the distance from the impurity position.

Important low-energy dynamical properties of a spin system can be measured in NMR experiments. The main NMR characteristics are the shift of the position of the NMR due to interactions, and the linewidth. The shift of NMR position is proportional to the magnetic susceptibility of the system. The NMR linewidth is related to the NMR relaxation rate. For the latter, $T_{1}^{-1}$, the main contribution from the position of the impurity in the spin chain can be presented as (cf. [7])

$$
T_{1}^{-1}=\left[\left(g^{\prime}\right)^{2} \mu_{B}^{2} \gamma_{N}^{2} / 2\right]\left[F_{0}^{z} S_{0}^{z z}\left(\omega_{N}\right)+F_{0}^{x} S_{0}^{x x}\left(\omega_{N}\right)\right],
$$

where $\gamma_{N}$ is the gyromagnetic ratio of the nuclear spin, $\omega_{N}$ is the resonance frequency of the nuclear spin, $F_{0}^{z, x}$ are hyperfine form-factors of the nuclear spin, parallel and perpendicular to the static magnetic field $H$ at the impurity site, $S_{0}^{\mu \nu}\left(\omega_{N}\right)(\mu \nu=x, z)$ are the components of the tensor of the dynamical structure factor of the spin chain at the impurity site, also parallel and perpendicular to $H$. The (resonance) frequency of the rf field in the NMR experiments is much smaller than the exchange interactions in the chain and the dc magnetic field, which acts on the electron spins of the chain, $\hbar \omega_{N} \ll J, J^{\prime}, g \mu_{B} H, g^{\prime} \mu_{B} H$ because of the difference between the electron and nuclear masses. The rotational symmetry of the system perpendicular to the dc field direction yields for the transverse components of the dynamical structure factor $S_{0}^{x x}=S_{0}^{y y}$. The fluctuation-dissipation theorem, see, e.g., [8], relates 
the dynamical structure factor with the imaginary part of the dynamical magnetic susceptibility $\chi^{\prime \prime}(\omega)$, which for our case yields

$$
S_{0}^{\mu v}\left(\omega_{N}\right)=\frac{2 \hbar \chi_{0}^{\prime \prime}\left(\omega_{N}\right)}{1-\exp \left(-\hbar \omega_{N} / k_{B} T\right)},
$$

where $T$ is the temperature, and $k_{B}$ is the Boltzmann's constant. It means that the NMR relaxation rate is proportional to the imaginary part of the local dynamical magnetic susceptibility of the impurity spin. For small $\hbar \omega_{N} \ll k_{B} T$, which is the usual case for NMR experiments in spin chain compounds, that relation can be rewritten as

$$
S_{0}^{\mu \nu}\left(\omega_{N}\right) \approx \frac{2 k_{B} T \chi_{0}^{\prime \prime}\left(\omega_{N}\right)}{\omega_{N}}
$$

First we calculate the longitudinal contribution to the dynamical structure factor at the impurity site as the Fourier transform of the spin-spin longitudinal autocorrelation function at the position of the impurity

$$
S_{0}^{z z}(\omega)=\int_{-\infty}^{\infty} d t\left\langle S_{0}^{z}(t) S_{0}^{z}(0)\right\rangle \mathrm{e}^{i \omega t},
$$

where the averaging is taken with the density matrix of the spin chain, and $S_{0}^{z}(t)=\exp (-i \mathcal{H} t / \hbar) S_{0}^{z}(0) \exp (i \mathcal{H} t / \hbar)$. Performing standard calculations (for this model we know all eigenvalues and eigenfunctions of the Hamiltonian exactly), we obtain

where

$$
S_{0}^{z z}(\omega)=2 \pi \delta(\omega)\left\langle S_{0}^{z}\right\rangle^{2}+A(\omega)
$$

$$
\begin{gathered}
A(\omega)=2 \pi \sum_{\lambda_{1}}\left|u_{0 \lambda_{1}}\right|^{2} \times \\
\times n_{\lambda_{1}} \sum_{\lambda_{2}}\left|u_{0 \lambda_{2}}\right|^{2}\left(1-n_{\lambda_{2}}\right) \delta\left(\left[\varepsilon_{\lambda_{1}}-\varepsilon_{\lambda_{2}}\right] / \hbar+\omega\right),
\end{gathered}
$$

$n_{\lambda}=\left[1+\exp \left(\varepsilon_{\lambda} / k_{B} T\right)\right]^{-1}$, and $\left\langle S_{0}^{z}\right\rangle$ is the average value of the $z$-projection of the spin moment at the impurity site at $t=0$. Taking into account that $\varepsilon_{2} \geq \varepsilon_{k} \geq \varepsilon_{1}$ it becomes clear that the contributions to $A(\omega)$ come from the states for which the following conditions exist: (i) The resonance of the local levels $\hbar \omega=\varepsilon_{2}-\varepsilon_{1}$, which contributes to $A(\omega)$ with the term $2 \pi u_{0,1}^{2} u_{0,2}^{2} n_{1}\left(1-n_{2}\right) \delta\left(\left[\varepsilon_{1}-\varepsilon_{2}\right] / \hbar+\omega\right)$; (ii) The resonance of the lower local level with the extended state $\hbar \omega=\varepsilon_{k_{1}}{ }^{*}-\varepsilon_{1}$, which contributes to $A(\omega)$ with the term $2 \pi u_{0,1}^{2} u_{0, k_{1}^{*}}^{2} n_{k_{1}^{*}}\left(1-n_{1}\right) \delta\left(\left[\varepsilon_{1}-\varepsilon_{k_{1}^{*}}\right] / \hbar+\omega\right)$; (iii) The resonance of the upper local level with the extended state $\hbar \omega=\varepsilon_{2}-\varepsilon_{k_{2}^{*}}$, which contributes to $A(\omega)$ with the term $2 \pi u_{0,2}^{2} u_{0, k_{2}^{*}}^{2} n_{2}\left(1-n_{k_{2}^{*}}\right) \delta\left(\left[\varepsilon_{k_{2}^{*}}-\varepsilon_{2}\right] / \hbar+\omega\right)$; finally (iv) the resonance of the extended levels $\hbar \omega=\varepsilon_{k_{2}}-\varepsilon_{k_{1}}$ with the contribution to $A(\omega)$ equal to

$$
2 \pi \sum_{k_{1}}\left|u_{0, k_{1}}\right|^{2}\left|u_{0, k_{2}}\right|^{2} n_{k_{1}}\left(1-n_{k_{2}}\right) \delta\left(\left[\varepsilon_{k_{1}}-\varepsilon_{k_{2}}\right] / \hbar-\omega\right) \text {. }
$$

Here the values of wave vectors $k_{1,2}^{*}$ are determined from the resonance conditions.

Since the nuclear magneton is much smaller than the electron one, one may take the limit $\omega_{N} \rightarrow 0$. In this limit the only (iv) process contributes to $A(0)$. Then the longitudinal component of the dynamical structure factor of the impurity can be written as

$$
\begin{gathered}
\alpha_{z z} \equiv S_{0}^{z z}(\omega=0)=2 \pi\left[\left\langle S_{0}^{z}\right\rangle^{2}+\frac{4 I^{4}}{\pi^{2}} \int_{-J}^{J} d y \times\right. \\
\times \frac{\exp \left[\left(y+g \mu_{B} H\right) / k_{B} T\right]}{\left(1+\exp \left[\left(y+g \mu_{B} H\right) / k_{B} T\right]\right)^{2}} \times \\
\left.\times \frac{\left(J^{2}-y^{2}\right)^{3 / 2}}{\left[4(J x-y)^{2}+4 I^{2} y(J x-y)+I^{4} J^{2}\right]^{2}}\right],
\end{gathered}
$$

where (c.f. [9])

$$
\begin{aligned}
\left\langle S_{0}^{z}\right\rangle & =\frac{1}{2}-\frac{2 I^{2}}{\pi} \int_{-J}^{J} \frac{d y}{1+\exp \left[\left(y+g \mu_{B} H\right) / k_{B} T\right]} \times \\
& \times \frac{\left(J^{2}-y^{2}\right)^{1 / 2}}{4(J x-y)^{2}+4 I^{2} y(J x-y)+I^{4} J^{2}}- \\
& -\frac{\left(1-r_{1}^{2}\right) \Theta\left(I^{2}-2 x-2\right)}{\left[1-r_{1}^{2}\left(I^{2}-1\right)\right]\left[1+\exp \left(\varepsilon_{1} / k_{B} T\right)\right]}- \\
& -\frac{\left(1-r_{2}^{2}\right) \Theta\left(I^{2}+2 x-2\right)}{\left[1-r_{2}^{2}\left(I^{2}-1\right)\right]\left[1+\exp \left(\varepsilon_{2} / k_{B} T\right)\right]},
\end{aligned}
$$

and $\Theta(x)$ is the Heaviside step function.

In the homogeneous spin chain in the ground state the quantum second order phase transition takes place at $H_{c}=J / g \mu_{B}$ [8]. At this value of the field the system undergoes the transition into the spin-polarized phase with the nominal value of the $z$-projection of the total moment, and the magnetic field behavior of the $z$-projection of the magnetic moment shows a cusp at $H=H_{c}$ in the ground state. The magnetic field behavior of the impurity spin is more complicated [9]. The magnitudes of the wave functions of the local levels have to be smaller than 1, which is the reason for the presence of the Heaviside step functions in the above expressions. Step functions can produce jumps (in the ground state) of the $z$-projection of the local magnetic moment of the impurity at the critical field $H_{0}=J I^{2} / 2 g \mu_{B} \sqrt{\gamma\left(I^{2}-\gamma\right)}$, where $\gamma=g^{\prime} / g$. Such a jump exists for $I^{2} \geq 2 \gamma$. For $I^{2}=2 \gamma$ the magnitude of the jump vanishes and we have $H_{0} \rightarrow H_{c}$ with the cusp in the field behavior of the $z$-projection of the impurity's magnetic moment in the ground state. Notice that for $\gamma<1$ the $z$-projection of the impurity's magnetic moment can be negative. 


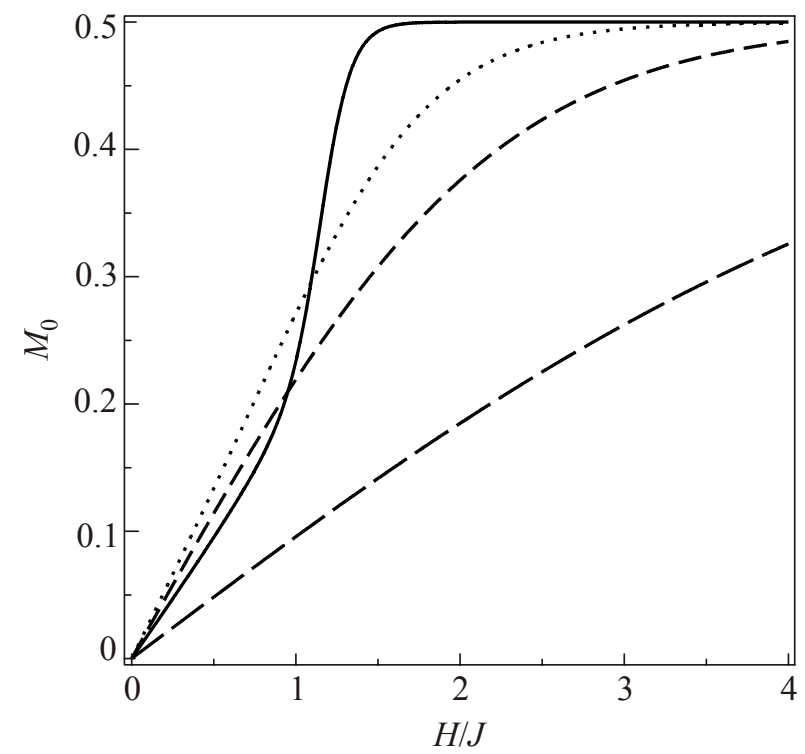

Fig. 1. The magnetic field dependence of the $z$-projection of the impurity's spin for $g^{\prime}=1.2, \quad I=2.2$. Lines show: solid $T=0.1 \mathrm{~J}$, dotted $T=0.5 \mathrm{~J}$, dashed $T=J$, long-dashed $T=3 \mathrm{~J}$.

The behavior of the $z$-projection of the impurity's spin $M_{0} \equiv\left\langle S_{0}^{z}\right\rangle$ is presented in Figs. 1 and 2. Notice that in all following figures we use units in which $k_{B}=\mu_{B}=J=g=1$. Figure 1 shows the field behavior of $M_{0}$ at $I=2.2$ and $g^{\prime}=1.2$ for several values of temperature. Temperature, as it must be, "smears out" the features related to the quantum phase transition (jump) at $H=H_{0}$. Figure 2 manifests the temperature behavior of $M_{0}$ for several values of the applied magnetic field.

Now let's turn to the results of calculations for the contribution of the longitudinal dynamical structure factor at the impurity site to the NMR relaxation rate $A(0)$. Figure 3

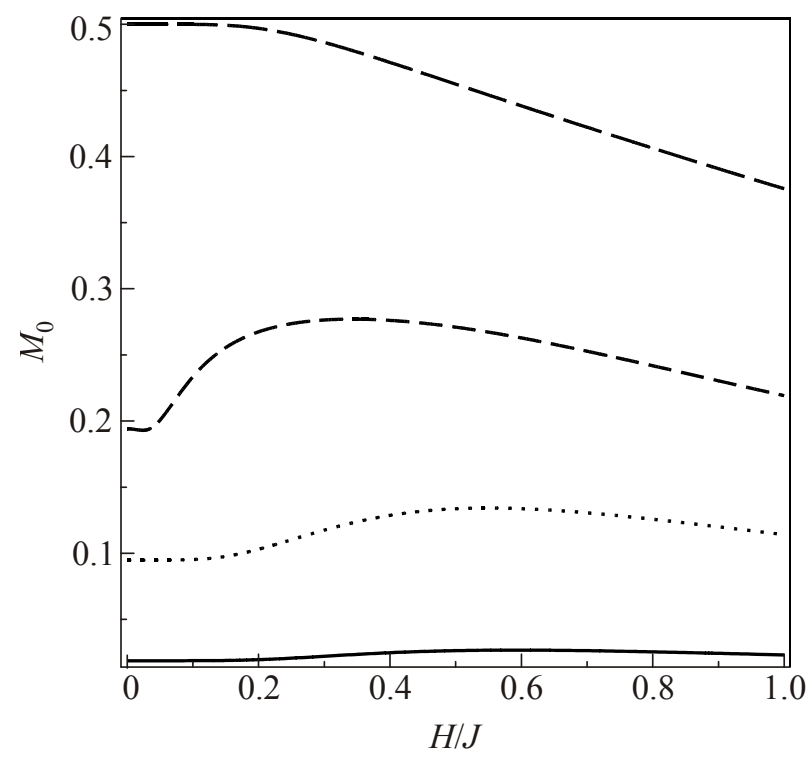

Fig. 2. The temperature dependence of the $z$-projection of the impurity's spin for $g^{\prime}=1.2, I=2.2$. Lines show: solid $H=0.1 J$, dotted $H=0.5 J$, dashed $H=J$, long-dashed $H=2 J$.

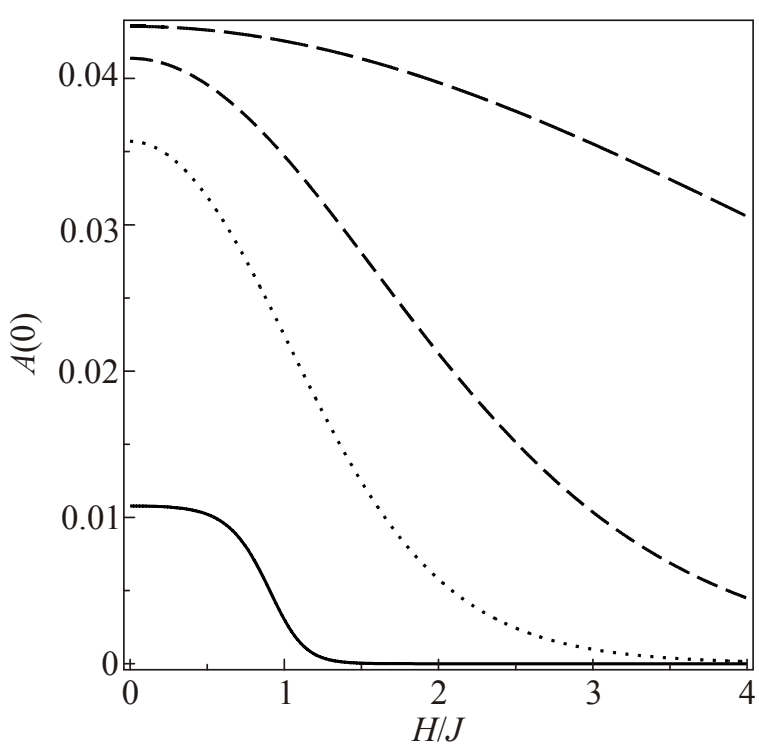

Fig. 3. The magnetic field dependence of the contribution to the longitudinal part of the dynamical structure factor of the impurity for $g^{\prime}=1.2, I=2.2$. Lines show: solid $T=0.1 J$, dotted $T=0.5 J$, dashed $T=J$, long-dashed $T=3 J$.

shows the magnetic field dependence of $A(0)$ for $g=1.2$ and $I=2.2$. For comparison we present results for the edge spin of the homogeneous spin chain in Fig. 4. It is clear that the main quantitative differences caused by the impurity are manifested at low field values at low temperatures.

The temperature dependence of $A(0)$ for the impurity and for the edge spin in the homogeneous chain are presented in Figs. 5 and 6. One can see that at low values of the field this contribution to the dynamical structure factor of the impurity is a convexing function the temperature,

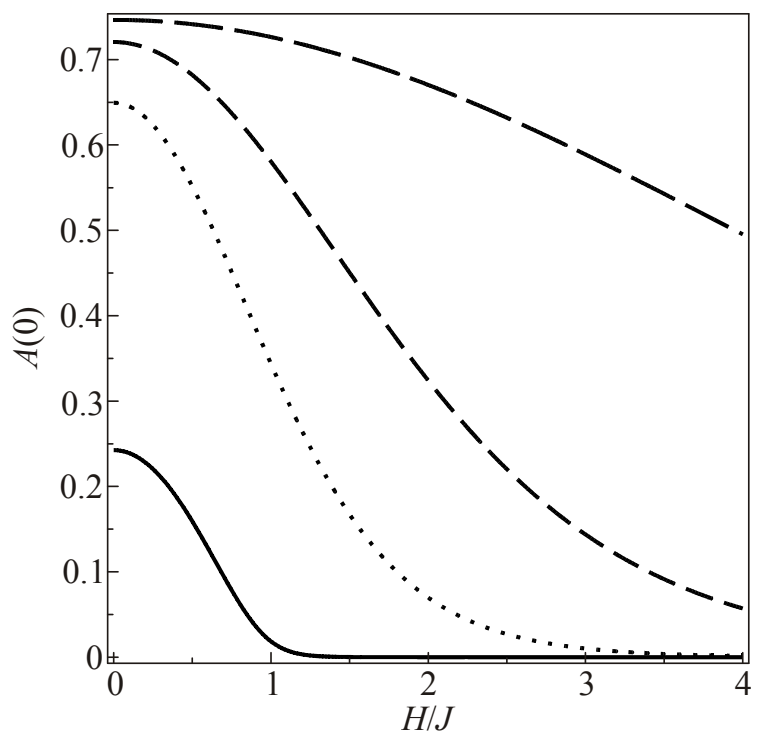

Fig. 4. The magnetic field dependence of the contribution to the longitudinal part of the dynamical structure factor of the edge spin in the homogeneous chain (for $\gamma=1, I=1$ ). Notations are the same as in Fig. 1. 


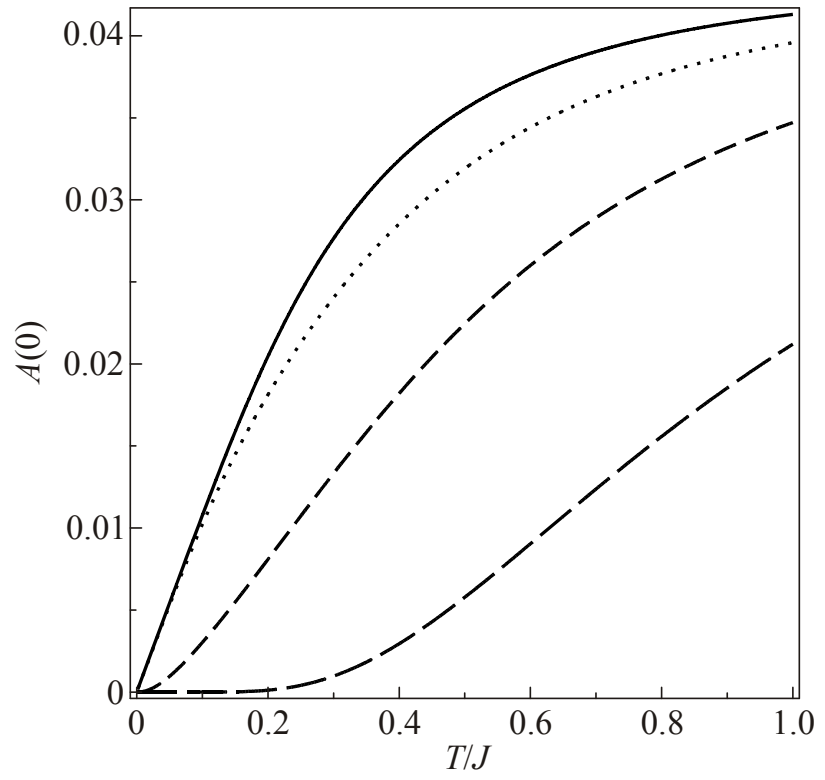

Fig. 5. The temperature dependence of the contribution to the longitudinal part of the dynamical structure factor of the impurity for $g^{\prime}=1.2, \quad I=2.2$. Lines show: solid $H=0.1 J$, dotted $H=0.5 J$, dashed $H=J$, long-dashed $H=2 J$.

while for larger values of $H>H_{0}$ it is a concaving function of $T$. Impurity again produces only quantitative changes to this characteristic.

Figures 7-9 present the dependencies of this contribution to the longitudinal dynamical structure factor of the impurity as the function of the relative strength of the impurity-host coupling $I$ (for $T=0.1 J$ at various values of

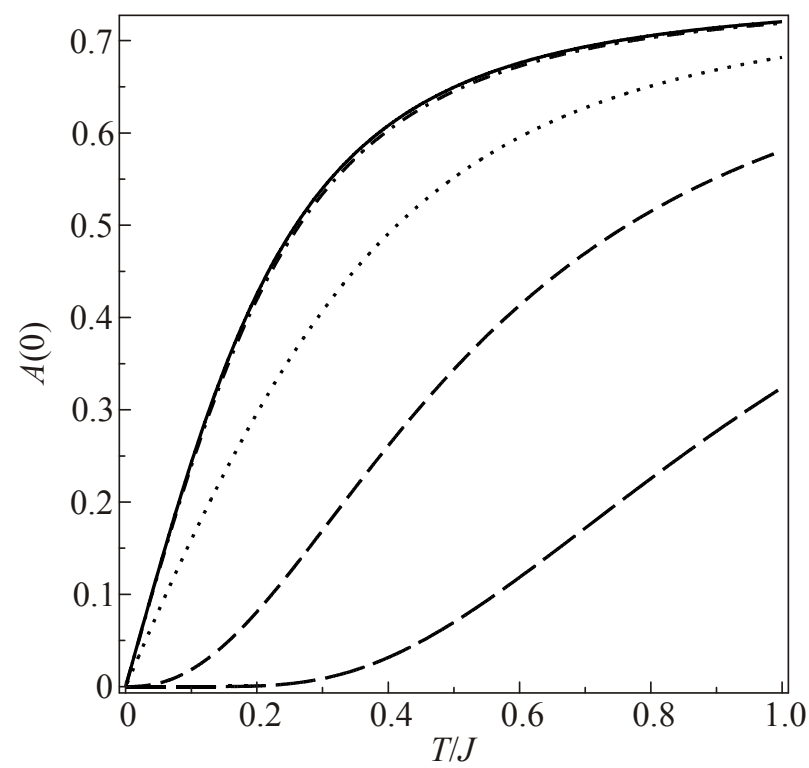

Fig. 6. The temperature dependence of the contribution to the longitudinal part of the dynamical structure factor of the edge spin in the homogeneous chain (for $\gamma=1, I=1$ ). Lines show: solid $H=0$, dashed-dotted $H=0.1 J$; dotted $H=0.5 J$, dashed $H=J$, long-dashed $H=2 J$.

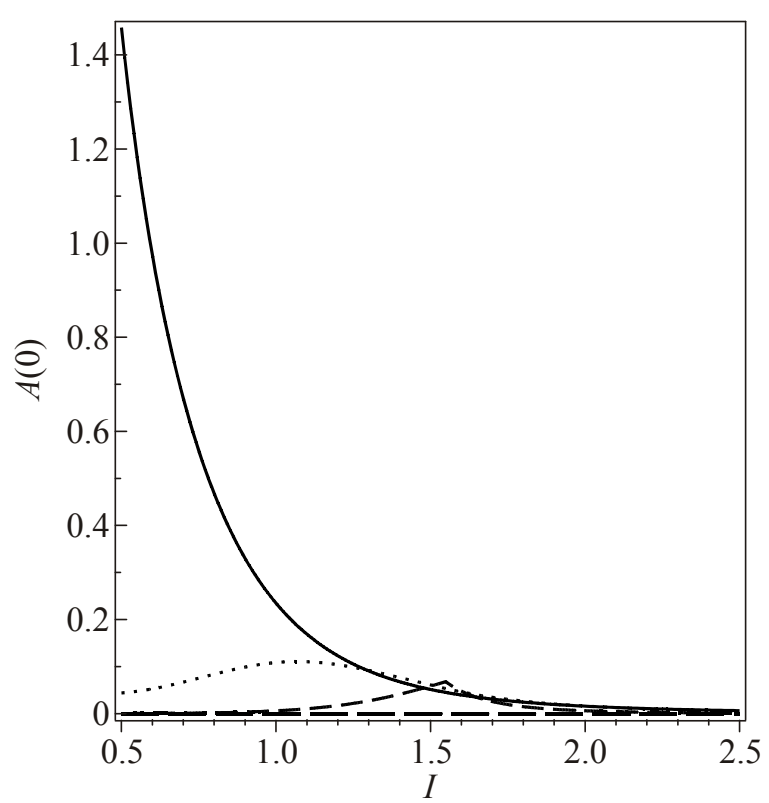

Fig. 7. The dependence of the contribution to the longitudinal part of the dynamical structure factor of the impurity for $g^{\prime}=1.2$ on the relative strength of the impurity-host coupling $I$ at $T=0.1 J$. Lines show: solid $H=0.1 J$, dotted $H=0.5 J$, dashed $H=J$, long-dashed $H=2 J$.

the field, Fig. 7, and for $H=0$ at various values of temperature, Fig. 8), and on the value of the effective $g$-factor of the impurity (Fig. 9). This contribution to the dynamical structure factor of the impurity decays with the growth of the impurity-host coupling for small values of the magnetic field, but it shows a maximum in $I$-dependence (for relatively small $I$ ) at higher fields. The features at $I^{2} \sim 2$

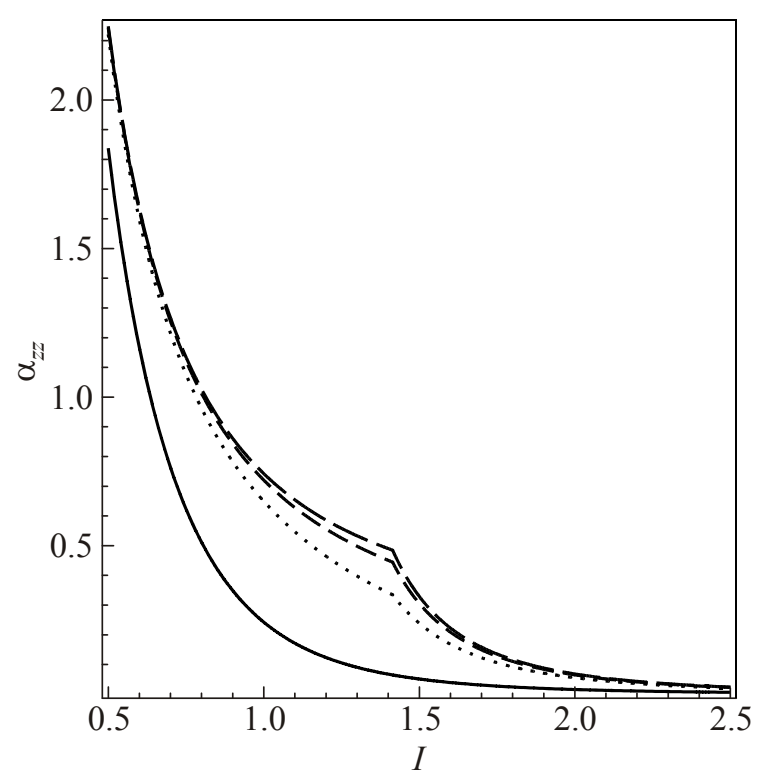

Fig. 8. The dependence of the longitudinal part of the dynamical structure factor of the impurity on the relative strength of the impurity-host coupling $I$ at $H=0$. Lines show: solid $T=0.1 J$, dotted $T=0.5 J$, dashed $T=J$, long-dashed $T=2 J$. 


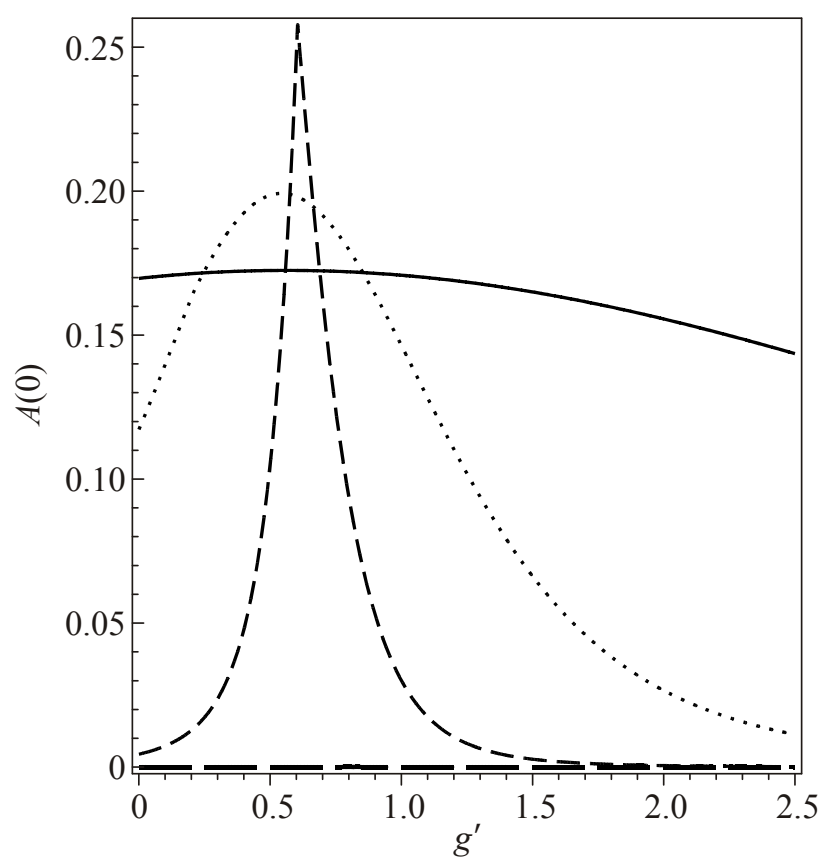

Fig. 9. The dependence of the contribution to the longitudinal part of the dynamical structure factor of the impurity for $I=1.1$ on the value of the impurity's effective $g$-factor $g^{\prime}$ at $T=0.1 J$. Lines show: solid $H=0.1 J$, dotted $H=0.5 J$, dashed $H=J$, long-dashed $H=2 J$.

in Fig. 8 at zero field (where $M_{0}=0$ ) are caused by the local impurity level. The nonmonotonic behavior in Fig. 9 takes place for intermediate values of $g^{\prime} \leq 1$ at intermediate values of the external field.

Now consider the contribution to the NMR relaxation rate, perpendicular to the dc magnetic field. Here we can use the results for the electron spin resonance of the impurity in a spin chain [10]. In the paper [10] the dynamical magnetic susceptibility of the impurity was calculated as the response to the $\mathrm{rf}$ magnetic field with the rotational polarization perpendicular to the dc field

$$
\chi_{0}^{\prime \prime}(\omega)=\pi \sum_{\lambda}\left|u_{0, \lambda}\right|^{2}\left(1-2 n_{\lambda}\right) \delta\left(\varepsilon_{\lambda}-\hbar \omega\right)
$$

We have

$$
\begin{gathered}
\chi_{0}^{\prime \prime}(\omega)=\tanh \left(\frac{\hbar \omega}{2 k_{B} T}\right)\left\{2 I^{2} \Theta\left(J-g \mu_{B} H+\hbar \omega\right) \times\right. \\
\times \sqrt{J^{2}-\left(g \mu_{B} H-\hbar \omega\right)^{2}} \times \\
\times\left[4\left(g^{\prime} \mu_{B} H-\hbar \omega\right)^{2}-4 I^{2}\left(g^{\prime} \mu_{B} H-\hbar \omega\right) \times\right. \\
\left.\times\left(g \mu_{B} H-\hbar \omega\right)+I^{4} J^{2}\right]^{-1}+ \\
+\pi \frac{I^{4} J^{2}-4\left(g^{\prime} \mu_{B} H-\hbar \omega\right)^{2}}{I^{4} J^{2}+4\left(I^{2}-1\right)\left(g^{\prime} \mu_{B} H-\hbar \omega\right)^{2}} \times \\
\left.\times\left[\delta\left(\varepsilon_{1}-\hbar \omega\right) \Theta\left(I^{2}-2 x-2\right)+\delta\left(\varepsilon_{2}-\hbar \omega\right) \Theta\left(I^{2}+2 x-2\right)\right]\right\} .
\end{gathered}
$$

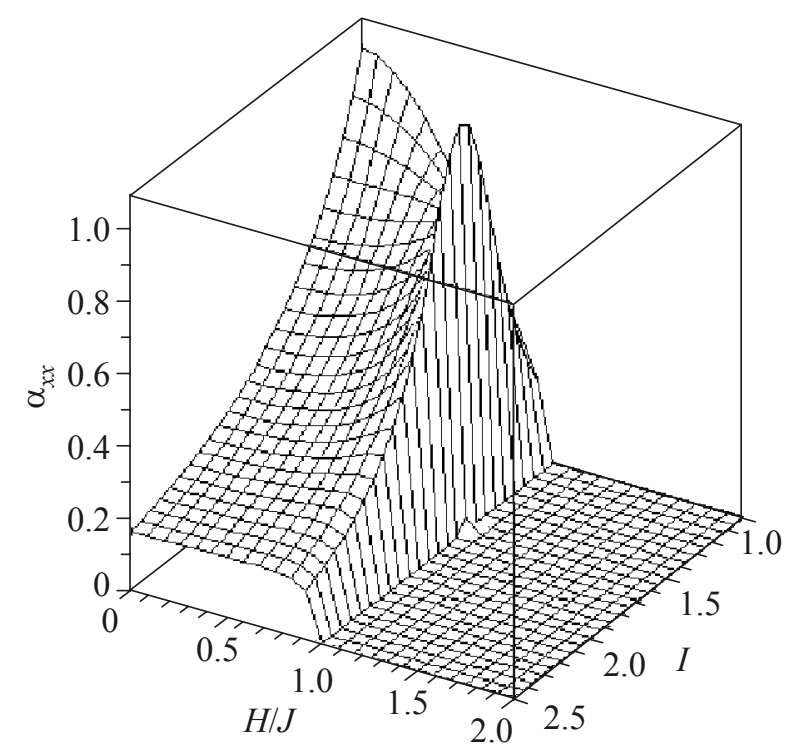

Fig. 10. The dependence of the transverse part of the dynamical structure factor of the impurity for $g^{\prime}=1.2$ on the relative strength of the impurity-host coupling $I$ and the external magnetic field.

The contribution to the NMR relaxation can be obtained via the component of the local dynamical structure factor, perpendicular to the dc magnetic field, as $S_{0}^{x x}(\omega=0)=$ $=\lim _{\omega_{N} \rightarrow 0} 2 k_{B} T \chi_{0}^{\prime \prime}\left(\omega_{N}\right) / \omega_{N}$. It turns out that for small $\omega_{N}$ this components of the dynamical structure factor for the impurity does not depend on the temperature. The magnetic field dependence of this component of the dynamical structure factor $\alpha_{x x} \equiv S_{0}^{x x}(0)$ are presented in Figs. 10 and 11.

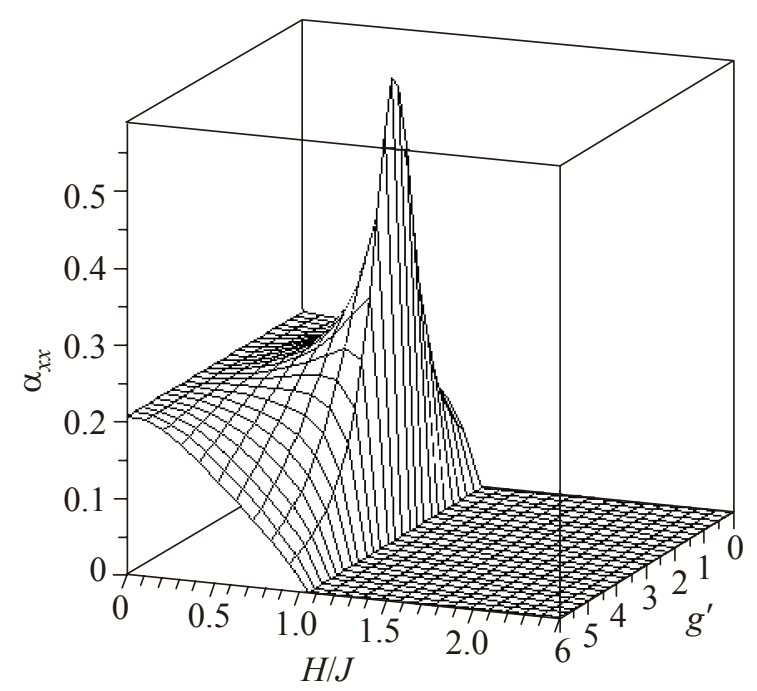

Fig. 11. The dependence of the transverse part of the dynamical structure factor of the impurity for $I=2.2$ on the effective $g$ factor of the impurity $g^{\prime}$ and the external magnetic field. 


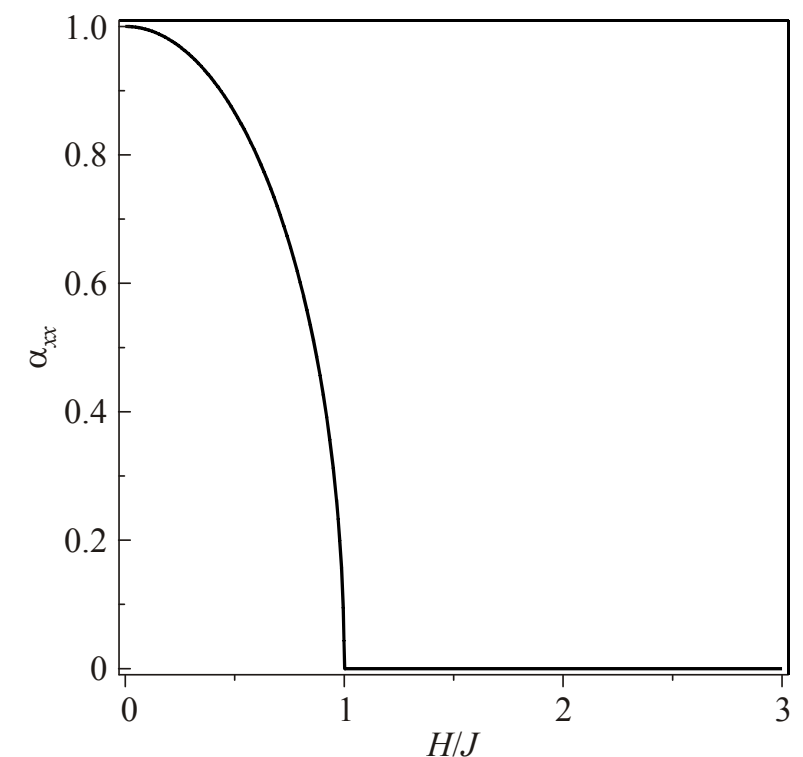

Fig. 12. The dependence of the transverse part of the dynamical structure factor of the edge spin of the homogeneous spin chain on the external magnetic field.

For comparison, Fig. 12 presents the magnetic field dependence of the transverse dynamical structure factor for the edge spin of the homogeneous spin chain, i.e., the case $I=\gamma=1$.

We can see that the magnetic field dependence of the transverse part of the dynamical strucrure factor of the impurity and the edge spin of the homogeneous chain decay with temperature (except of special regions of $I$ and $g^{\prime}$ ) and becomes zero at $H \geq H_{c}$. On the other hand, from Figs. 10 and 11 it is clear that in the regions $I^{2} \sim 2$ and $\gamma \sim 1$ the magnetic field dependence of the transverse component of the dynamical structure factor of the impurity is non-monotonic.

In summary, in this paper we have investigated how the NMR relaxation rate of the spin chain with a magnetic impurity depends on the parameters, which distinguish the impurity: the local exchange interaction between the impurity and the host chain and the effective $g$-factor of the impurity. We have shown that the temperature and the magnetic field dependencies of the NMR relaxation are strongly affected by the impurity. In particular, the field dependence of the NMR relaxation rate at the impurity site can manifest jump-like features at low temperatures, caused by local levels. On the other hand, the temperature dependencies of the NMR relaxation can show nonmonotonic behavior caused by the impurity. We believe that such features are characteristic for the NMR in spin chains with magnetic impurities, and they can be observed in NMR experiments in organic and nonorganic quasi-onedimensional magnetic materials.

I thank MPI PKS Dresden for kind hospitality, and H.-H. Klauss and H. Kühne (TU Dresden) for helpful discussions of the obtained results. The support from the Institute for Chemistry of V.N. Karazin Kharkov National University is acknowledged.

I dedicate this work to Academician Professor Viktor Valentinovich Eremenko, who has suggested me to study theoretical physics, and who has done so much for me, on the occasion of his 80th birthday.

1. For the review see, A.A. Zvyagin, Finite Size Effects in Correlated Electron Models: Exact Results, Imperial College Press, London (2005) and references therein.

2. F. Borsa and M. Mali, Phys. Rev. B9, 2215 (1974); M. Takigawa, N. Motoyama, H. Eisaki, and S. Uchida, Phys. Rev. Lett. 76, 4612 (1996); G. Chaboussant, Y. Fagot-Revurat, M.-H. Julien, M.E. Hanson, C. Berthier, M. Horvatić, L.P. Lévy, and O. Piovesana, Phys. Rev. Lett. 80, 2713 (1998); K.R. Thurber, A.W. Hunt, T. Imai, and F.C. Chou, Phys. Rev. Lett. 87, 247202 (2001); A.U.B. Wolter, P. Wzietek, S. Süllow, F.J. Litterst, A. Honecker, W. Brenig, R. Feyerherm and H.-H. Klauss, Phys. Rev. Lett. 94, 057204 (2005); H. Kühne, H.-H. Klauss, S. Grossjohann, W. Brenig, F.J. Litterst, A.P. Reyes, P.L. Kuhns, M.M. Turnbull, and C.P. Landee, Phys. Rev. B80, 045110 (2009); H. Kühne, A.A. Zvyagin, M. Günther, A.P. Reyes, P.L. Kuhns, M.M. Turnbull, C.P. Landee, and H.-H. Klauss, Phys. Rev. B83, 100407(R) (2011).

3. T. Yamada, Z. Hiroi, and M. Takano, J. Solid State Chem. 156, 101 (2001); T. Yamada, M. Takano, and Z. Hiroi, J. Alloys Comp. 317-318, 171 (2001); A.A. Zvyagin, Fiz. Nizk. Temp. 32, 214 (2006) [Low Temp. Phys. 32, 158 (2006)].

4. F. Hammerath, S. Nishimoto, H.-J. Grafe, A.U.B. Wolter, V. Kataev, P. Ribeiro, C. Hess, S.-L. Drechsler, and B. Büchner, Phys. Rev. Lett. 107, 017203 (2011).

5. P. Jordan and E. Wigner, Z. Phys. 47, 631 (1928).

6. A.A. Zvyagin, Fiz. Nizk. Temp. 38, 266 (2012) [Low Temp. Phys. 38, 210 (2012)].

7. T. Moriya, Progr. Theor. Phys. 16, 23 (1956).

8. See, e.g., A.A. Zvyagin, Quantum Theory of One-Dimensional Spin Systems, Cambridge Scientific Publishers, Cambridge (2010).

9. V.Z. Kleiner and V.M. Tsukernik, Fiz. Nizk. Temp. 6, 332 (1980) [Sov. J. Low Temp. Phys. 6, 158 (1980)].

10. V.Z. Kleiner and V.M. Tsukernik, Fiz. Met. Metalloved. 39, 947 (1975). 